\title{
NO PRODUCTION OF ENTROPY IN THE EULER FLUID
}

\author{
R. F. STREATER \\ Department of Mathematics, King's College London \\ Strand, London WC2R 2LS, UK \\ E-mail: raymond.streater@kcl.ac.uk
}

\begin{abstract}
We derive the Euler equations as the hydrodynamic limit of a stochastic model of a hard-sphere gas. We show that the system does not produce entropy.
\end{abstract}

1. Hydrostatics of a gas of hard spheres. We take space to be $\Lambda \subseteq(a \mathbf{Z})^{3}$, and suppose the length $a$, representing the diameter of a molecule, to be so small compared with the variation of the macroscopic fields that we can replace all sums over $\Lambda$ by integrals. The possible configurations of the fluid are the points in the product sample space

$$
\Omega=\prod_{x \in \Lambda} \Omega_{\boldsymbol{x}}
$$

so a configuration is specified by the collection $\left\{\omega_{\boldsymbol{x}}\right\}_{\boldsymbol{x} \in \Lambda}$. For each $\boldsymbol{x}$,

$$
\Omega_{\boldsymbol{x}}=\left\{\emptyset,(\epsilon \mathbf{Z})^{3}\right\} .
$$

Here, $\epsilon$ is a small parameter having the dimension of momentum. If the system is in a configuration $\omega$, such that $\omega_{\boldsymbol{x}}=\emptyset$, then we say that the site $\boldsymbol{x}$ is empty. If $\omega_{\boldsymbol{x}}=\boldsymbol{k}$, we say that the site $\boldsymbol{x}$ is occupied, by a particle of momentum $\boldsymbol{k}$. This simple exclusion of more than one particle on each site incorporates the hard-core repulsion between the particles, which are thus hard spheres sitting at some of the points of $\Lambda$.

The state of the system is a probability on $\Omega$, denoted by $\mu$. We denote the set of states by $\Sigma$. The 'slow variables' of our model are the 5 extensive conserved random fields

$$
\begin{aligned}
& \mathcal{N}_{\boldsymbol{x}}(\omega)= \begin{cases}0 & \text { if } \omega_{\boldsymbol{x}}=\emptyset, \\
1 & \text { if } \omega_{\boldsymbol{x}}=\boldsymbol{k},\end{cases} \\
& \mathcal{E}_{\boldsymbol{x}}(\omega)= \begin{cases}0 & \text { if } \omega_{\boldsymbol{x}}=\emptyset, \\
\boldsymbol{k} \cdot \boldsymbol{k} / 2 m+\Phi(\boldsymbol{x}) & \text { if } \omega_{\boldsymbol{x}}=\boldsymbol{k},\end{cases}
\end{aligned}
$$

2000 Mathematics Subject Classification: 35Q20, 76B, 82D15.

Key words and phrases: entropy, Euler equations.

The paper is in final form and no version of it will be published elsewhere. 


$$
\mathcal{P}_{\boldsymbol{x}}(\omega)= \begin{cases}0 & \text { if } \omega_{\boldsymbol{x}}=\emptyset \\ \boldsymbol{k} & \text { if } \omega_{\boldsymbol{x}}=\boldsymbol{k}\end{cases}
$$

Here, $\Phi(\boldsymbol{x})$ is the external potential energy per particle. The slow variables appearing in hydrodynamics are the $n=5|\Lambda|$ means in the state $\mu$ :

$$
N_{\boldsymbol{x}}=\mathbf{E}_{\mu}\left[\mathcal{N}_{\boldsymbol{x}}\right] ; \quad E_{\boldsymbol{x}}=\mathbf{E}_{\mu}\left[\mathcal{E}_{\boldsymbol{x}}\right] ; \quad \Pi_{\boldsymbol{x}}=\mathbf{E}_{\mu}\left[\mathcal{P}_{\boldsymbol{x}}\right] .
$$

The (von Neumann) entropy of any state $\mu$ is

$$
S(\mu):=-k_{B} \sum_{\omega} \mu(\omega) \log \mu(\omega) .
$$

In information geometry, the choice of slow variables $\left\{X_{1}, X_{2} \ldots, X_{n}\right\}$ defines the information manifold $\mathcal{M}$, which consists of states of maximum entropy among all states with given means, say

$$
\mathbf{E}_{\mu}\left[X_{j}\right]:=\mu \cdot X_{j}=\eta_{j}, \quad j=1, \ldots, n ;
$$

By the Gibbs-Jaynes principle, such a state has the form

$$
\mu(\omega)=\exp \left\{-\sum_{j=1}^{n} \xi_{j} X_{j}(\omega)-\log \Xi\right\}
$$

where the dual, or canonical, variables $\xi_{j}$ are Lagrange multipliers determined uniquely by the means $\eta_{i}$. In our case, for each $\boldsymbol{x}$, the duals to energy, number, and momentum are, respectively, $\beta_{\boldsymbol{x}}, \xi_{\boldsymbol{x}}, \boldsymbol{\zeta}_{\boldsymbol{x}}$, and so the state has the form

$$
\mu(\omega)=\prod_{\boldsymbol{x}} \Xi_{\boldsymbol{x}}^{-1} \exp \left\{-\xi_{\boldsymbol{x}} \mathcal{N}_{\boldsymbol{x}}(\omega)-\beta_{\boldsymbol{x}} \mathcal{E}_{\boldsymbol{x}}(\omega)-\boldsymbol{\zeta}_{\boldsymbol{x}} \cdot \mathcal{P}_{\boldsymbol{x}}(\omega)\right\} .
$$

Such a state is said to be in local thermodynamic equilibrium, LTE. Equilibrium holds when $\beta \ldots$ are independent of $\boldsymbol{x}$. In [7] we found the (grand) partition function at point $\boldsymbol{x}$,

$$
\Xi_{\boldsymbol{x}}=1+\left(\frac{2 \pi m}{\epsilon^{2} \beta_{\boldsymbol{x}}}\right)^{3 / 2} \exp \left\{-\xi_{\boldsymbol{x}}-\beta_{\boldsymbol{x}} \Phi(\boldsymbol{x})+m \boldsymbol{\zeta}_{\boldsymbol{x}} \cdot \boldsymbol{\zeta}_{\boldsymbol{x}} / 2 \beta_{\boldsymbol{x}}\right\}
$$

by replacing the sum over the momentum lattice of size $\epsilon$ by a Gaussian integral. The product structure of an $L T E$ state means that an observable at a point of $\Lambda$ is independent of an observable at any other. Note that $\mu_{\boldsymbol{x}}\{\emptyset\}=\left(1-N_{\boldsymbol{x}}\right)$. It then follows from (2) that

$$
1-N_{\boldsymbol{x}}=\Xi^{-1} \text {. }
$$

If $\omega_{\boldsymbol{x}} \neq \emptyset$, the state $\mu$ can be written in Maxwell form

$$
\mu(\boldsymbol{x}, \boldsymbol{k})=N_{\boldsymbol{x}} p(\boldsymbol{x}, \boldsymbol{k})=N_{\boldsymbol{x}} Z^{-1} \exp \left\{-\beta_{\boldsymbol{x}} \Phi(\boldsymbol{x})-\beta_{\boldsymbol{x}} \boldsymbol{k} \cdot \boldsymbol{k} /(2 m)-\boldsymbol{\zeta}_{\boldsymbol{x}} \cdot \boldsymbol{k}\right\}
$$

where $Z$ is the canonical partition function:

$$
Z_{x}=\left(\frac{2 \pi m}{\epsilon^{2} \beta_{\boldsymbol{x}}}\right)^{3 / 2} \exp \left\{-\beta_{\boldsymbol{x}} \Phi(\boldsymbol{x})+\frac{m \boldsymbol{\zeta}_{\boldsymbol{x}} \cdot \boldsymbol{\zeta}_{\boldsymbol{x}}}{2 \beta_{\boldsymbol{x}}}\right\}
$$

We note the identity for each $\boldsymbol{x}$

$$
\Xi=1+e^{-\xi} Z=1+e^{-\xi-\beta \Phi} Z_{0}
$$


where $Z_{0}$ is the canonical partition function when $\Phi=0$. The external potential does not influence the local velocity distribution $p$, as it is cancelled out by the partition function. The mean fields are related to the canonical fields by

$$
\begin{aligned}
E_{\boldsymbol{x}} & =-\frac{\partial}{\partial \beta_{\boldsymbol{x}}} \log \Xi_{\boldsymbol{x}}=N(\boldsymbol{x})\left(\Phi(\boldsymbol{x})+\frac{3}{2 \beta_{\boldsymbol{x}}}+\frac{m \boldsymbol{\zeta}_{\boldsymbol{x}} \cdot \boldsymbol{\zeta}_{\boldsymbol{x}}}{2 \beta_{\boldsymbol{x}}^{2}}\right) \\
N_{\boldsymbol{x}} & =-\frac{\partial}{\partial \xi_{\boldsymbol{x}}} \log \Xi_{\boldsymbol{x}}=\frac{\Xi_{\boldsymbol{x}}-1}{\Xi_{\boldsymbol{x}}}=\frac{Z e^{-\xi_{\boldsymbol{x}}}}{1+Z e^{-\xi_{\boldsymbol{x}}}} \\
\Pi_{\boldsymbol{x}}^{i} & =-\frac{\partial}{\partial \zeta_{i}} \log \Xi_{\boldsymbol{x}}=-\frac{m N_{\boldsymbol{x}} \zeta_{\boldsymbol{x}}^{i}}{\beta_{\boldsymbol{x}}}
\end{aligned}
$$

The hydrodynamic variables are the mass-density $\rho=m N^{-3}$, the velocity field $\boldsymbol{u}=-\boldsymbol{\zeta} / \beta$, the temperature $\Theta=\left(k_{B} \beta\right)^{-1}$, and the potential energy per unit mass, $\phi=\Phi / m$. We shall therefore eliminate $\xi, \beta, \zeta^{j}$ in favour of $\rho, \Theta, u^{j}$. Only $\xi$ remains to be found:

$$
e^{-\xi \boldsymbol{x}}=Z_{\boldsymbol{x}}^{-1} N_{\boldsymbol{x}} /\left(1-N_{\boldsymbol{x}}\right)
$$

or

$$
\xi=-\beta \Phi-\log N+\log (1-N)+\log Z_{0}
$$

where $Z_{0}$ is the canonical partition function when $\Phi=0$. We need its gradient:

$$
\partial_{j} \xi=\frac{3 \partial_{j} \Theta}{2 \Theta}-\frac{m}{2 k_{B}} \frac{u^{i} u^{i}}{\Theta^{2}} \partial_{j} \Theta+\frac{m u^{i}}{k_{B} \Theta} \partial_{j} u^{i}-\frac{\partial_{j} N}{N}-\frac{\partial_{j} N}{1-N}-\frac{\partial_{j} \Phi}{k_{B} \Theta}+\frac{\Phi}{k_{B}} \frac{\partial_{j} \Theta}{\Theta^{2}} .
$$

We denote by $E, N$ and $\Pi$ the total values of the mean energy, number and momentum; then (1) gives for the entropy at equilibrium

$$
\Theta S(\mu)=E+k_{B} \Theta \xi N-\boldsymbol{u} \cdot \boldsymbol{\Pi}+k_{B} \Theta \log \Xi .
$$

Compare this with the thermostatic formula

$$
\Theta S=E+k_{B} \Theta \xi N-\boldsymbol{u} \cdot \boldsymbol{\Pi}+P V
$$

where $P$ is the pressure and $V$ is the volume; we see that

$$
P=k_{B} \frac{|\Lambda|}{V} \Theta \log \Xi=-k_{B} \Theta a^{-3} \log (1-N) .
$$

2. The fundamental equation. Our model for the dynamics is a path $\{\mu(t)\}_{t \geq 0}$ through $\Sigma(\Omega)$ determined by the Liouville motion, interrupted by a thermalization at random points $\boldsymbol{x}$, occurring at random times. After a thermalization at $\boldsymbol{x}$, the state changes to one in which observables at $\boldsymbol{x}$ are statistically independent of those at any $\boldsymbol{y} \neq \boldsymbol{x}$, and, restricted to $\Omega_{\boldsymbol{x}}$, the state is in thermodynamic equilibrium. The law for the random time between collisions is of exponential form, but the rate of the process depends on the local density of the gas, and its temperature, and so on the state itself. This means that the dynamics falls outside the usual theory of Markov chains, in which the updated state is linear in the current state, and the transition matrix is the same for all states; we are in the business of non-linear Markov chains. Nonlinearity itself is not the problem; the ultimate goal of this work is to put an external potential $\Phi$ into our version [7] of the Navier-Stokes equations, which are non-linear. However, we are going to use the concept of conditional probability to derive the master equation, and care is 
needed if we stray from a path in $\Omega$ to a path in $\Sigma(\Omega)$ which does not come from a path in $\Omega$. We shall adopt the following way out, which makes use of the assumption that the density is low, and so the collision probability is small. In calculating the probability per unit time that a configuration $\omega$ at time $t \in\left(t_{0}-t, t_{0}\right)$ move to another point of $\Omega$, we shall assume that the hydrodynamic parameters satisfies the Euler equations, (30)-(32) in the small time interval $\left(t_{0}-T, t_{0}\right)$. The initial values of the hydrodynamic parameters in these equations are taken to be those of the true state at time $t_{0}-T$. Here we only need to consider $T<20 t_{\ell}$, since the survival probability falls exponentially. We then assume that the Markov process between $t_{0}-T$ and $t_{0}$ is linear as usual, and takes place in an ambient Euler fluid which determines the rate of thermalization. In this paper, we show that, neglecting collisions, the means of the slow variables do indeed satisfy the Euler equations, showing self-consistency. We can then show that there is no production of entropy in this case.

The Liouville dynamics is determined as follows. If at time $t=0$ a configuration $\omega \in \Omega$ has a particle at $\boldsymbol{x}$, then it follows Newton's laws

$$
\frac{d \boldsymbol{x}}{d t}=\frac{\boldsymbol{k}}{m}, \quad \frac{d \boldsymbol{k}}{d t}=-\nabla \Phi:=m \boldsymbol{f}
$$

for a time. Before we take the continuum limit, space is $\Lambda$, which is discrete; a particle following (11) will nearly always leave the lattice. In that case, after any given random time $t$ after which a thermalization occurs, we place the resulting thermalized particle at the lattice site nearest to $\boldsymbol{x}(t)$, ties being decided by tossing a coin. In the limit $a \rightarrow 0$ we expect this to introduce a negligible error in the location being assigned to the particle.

Let $\mu \in \Sigma(\Omega)$ and define $N_{\boldsymbol{x}}=\mu\left\{\omega_{\boldsymbol{x}} \neq \emptyset\right\}$, the probability that $\boldsymbol{x}$ is occupied in the state $\mu$. Then $N_{\boldsymbol{x}}=\mu \cdot \mathcal{N}_{\boldsymbol{x}}$. Let $p_{\boldsymbol{x}}(\boldsymbol{k})$ be the conditional probability that $\omega_{\boldsymbol{x}}=\boldsymbol{k}$, given that $\mathcal{N}_{\boldsymbol{x}}=1$ :

$$
p_{\boldsymbol{x}}(\boldsymbol{k})=\mu\left\{\omega: \omega_{\boldsymbol{x}}=\boldsymbol{k} \mid \omega_{\boldsymbol{x}} \neq \emptyset\right\}=N_{\boldsymbol{x}}^{-1} \mu\left\{\omega: \omega_{\boldsymbol{x}}=\boldsymbol{k}\right\}
$$

Thus,

$$
\mu\left\{\omega: \omega_{\boldsymbol{x}}=\boldsymbol{k}\right\}=N_{\boldsymbol{x}} p(\boldsymbol{x}, \boldsymbol{k}) .
$$

This does not mean of course that $\mathcal{N}_{\boldsymbol{x}}$ and $\mathcal{P}_{\boldsymbol{x}}$ are independent in the state $\mu$.

Given that no collisions occur, the particles obey (11), and this induces the Liouville motion on the states, namely, Boltzmann's equation with no collision term:

$$
\frac{\partial \mu(\boldsymbol{x}, \boldsymbol{k})}{\partial t}+\frac{\boldsymbol{k}}{m} \cdot \boldsymbol{\partial} \mu(\boldsymbol{x}, \boldsymbol{k})+\boldsymbol{f} \cdot \nabla_{\boldsymbol{k}} \mu(\boldsymbol{x}, \boldsymbol{k}, t)=0 .
$$

This dynamics of the probabilities does not correspond to an underlying motion in $\Omega$ for all time. We can find initial conditions for which two particles both arrive within a distance $a / 2$ from the same point at the same time. Such a configuration does not lie in $\Omega$. Our assumptions of no collisions is true with high probability for a few time steps, but collisions are almost sure to occur eventually. We will replace the problem of tracking which collisions actually occur if we follow (11) by Boltzmann's idea of introducing a probability for collisions. Instead of giving, as Boltzmann did, the probability that a pair of particles with given position and momentum produce another specified pair, it will be enough to assume that the collision is $100 \%$ efficient (in the terminology of [6]). 
This means that after a collision at $x$, of a particle with momentum $k$, we replace this configuration by a particle at $\boldsymbol{x}$ with momentum $\boldsymbol{k}^{\prime}$, with probability determined by the Maxwell distribution of mean momentum $\boldsymbol{k}$ and mean energy $\boldsymbol{k} \cdot \boldsymbol{k} /(2 m)+\Phi$. By construction, this does not alter the means of the slow variables. We shall refer to this event as a thermalization, rather than as a collision. We shall assume that the mean free path is large on the molecular scale, and neglect the possibility that a snapshot of the lattice catch a particle in the process of thermalizing: with high probability it will be in Newtonian motion between collisions. From any initial configuration $\omega$, we can follow the dynamics as time progresses, following this dynamics. It is a random path through $\Omega$, that is, a process, defined for $t_{0}-T \leq s \leq t_{0}$, and described by the family of points $\{\mu(s)\}_{t_{0}-T \leq s \leq t_{0}}$.

Let $t$ denote the random time between collisions, whose law is determined by the conditional probability $w\left(\boldsymbol{x}, \boldsymbol{k}, t_{0} ; t\right) d t$ that a particle, certainly at $\boldsymbol{x}$ at time $t_{0}$ with momentum $\boldsymbol{k}$, travel under Newton's laws a free time $t$ and thermalize in the interval $\left(t_{0}+t, t_{0}+t+d t\right)$. Since it will thermalize eventually,

$$
\int_{0}^{\infty} w\left(\boldsymbol{x}, \boldsymbol{k}, t_{0} ; t\right) d t=1 .
$$

The important small parameter is the mean free time, also called the relaxation time, $t_{\ell}$ :

$$
t_{\ell}\left(\boldsymbol{x}, \boldsymbol{k}, t_{0}\right):=\int_{0}^{\infty} t w\left(\boldsymbol{x}, \boldsymbol{k}, t_{0} ; t\right) d t .
$$

This is invariant under $\mathcal{G}$, unlike the mean free path, which is related by $\ell=\boldsymbol{k} t_{\ell} / m$. For free particles, $\Phi=0$, these were found in [7]. They are not affected by the potential, so we use the same values here. We also introduced

$$
\begin{aligned}
C(\boldsymbol{y}, \boldsymbol{k}, t) d t & =\operatorname{Prob}\{\text { a particle at }(\boldsymbol{y}, \boldsymbol{k}) \text { at time } t \text { thermalize in }(t, t+d t)\} \\
W\left(\boldsymbol{x}, \boldsymbol{k}, t_{0} ; t\right) & =\operatorname{Prob}\left\{\text { a particle at }\left(\boldsymbol{x}, \boldsymbol{k}, t_{0}\right) \text { survive up to time } t_{0}+t\right\} .
\end{aligned}
$$

Put $z(t):=(\boldsymbol{x}(t), \boldsymbol{k}(t)):=\tau_{t}(z)$, the solution to Newton's equations with initial point $z:=(\boldsymbol{x}, \boldsymbol{k})$. Then we have the relation

$$
w\left(\boldsymbol{x}, \boldsymbol{k}, t_{0} ; t\right)=W\left(\boldsymbol{x}, \boldsymbol{k}, t_{0} ; t\right) C\left(z(t), t+t_{0}\right)
$$

and just as in [7] we get from (15),

$$
W\left(\boldsymbol{x}, \boldsymbol{k}, t_{0} ; t\right)=\exp \left\{-\int_{0}^{t} C\left(z\left(t_{1}\right), t_{0}+t_{1}\right) d t_{1}\right\} .
$$

As in [7], eq. (36), $C$ is proportional to the density $\rho$. Thus if $\rho$ is bounded below by a positive number, we get an exponential decrease for $W$, the survival probability, along an orbit.

The fundamental equation will relate the probability distribution $\mu\left(z, t_{0}\right)$ at an arbitrary point $z$ in phase space at the time $t_{0}$ to a Maxwell distribution $\bar{\mu}$ at the same point, with small correction terms. Let $z(s)$ denote (for $s>0$ ) the point in phase space on the backward Newtonian orbit of the point $z$ at time $t_{0}-s$. For each sample path $\omega(\cdot)$, in which $z$ is occupied at time $t_{0}$, there is a unique time $t_{0}-t$ at which the last thermalization occurred, in that no further thermalizations took place on the orbit $\{z(s)\}_{0<s<t}$. 
It follows that the free time of the particle thermalized at $t_{0}-t$ must have a free time $t^{\prime}$ say, with $t^{\prime}>t$.

In the following, 'event' will mean an event in the sample space (the path space of $\Omega$ ) of the process constructed above in the time interval $\left(t_{0}-T, t_{0}\right)$. Let $E(s)$ denote the event, that there is a particle at $z(s)$ at time $t_{0}-s$. We shall consider the conditional probabilities of collision and free motion along the orbit, given $E(0)$. We shall then recover a formula for $\mu(z(0))$ using Bayes's theorem. Liouville's theorem, that $d^{3} x d^{3} k$ is invariant under Newton's laws, enables us to deduce equations relating the density of probability from equations relating probabilities. For example we shall write $\operatorname{Pr}\{E(0)\}=\mu\left(\boldsymbol{x}, \boldsymbol{k}, t_{0}\right)$, referring to the densities.

Let $F(t)$ be the event, that $E(0)$ occurred, and the last collision occurred at time $t_{0}-t$ producing a particle at the phase point $z(t)$. Then $F(t) \subseteq E(0)$, since the event produced exactly the right state, $z(t)$, which evolves to our point $z(0)$, as it undergoes no further collisions. Let $F(a, b)$ denote the event, that the last collision was at some $s, a \leq s \leq b$. If $(a, b) \cap(c, d)=\emptyset$, then $F(a, b)$ and $F(c, d)$ are disjoint. So, assuming smoothness, $F(t)$ has a probability density on $\mathbf{R}^{+}$, say $f(t)$. Let $G\left(t, t^{\prime}\right)$ be the event that $z(t)$ is occupied at time $t_{0}-t$, and that the particle's free time is $t^{\prime}$. Let $H\left(t_{1}\right)$ be the event that $F(s)$ occurred for some $s<t_{1}$. We shall need the formula

$$
\begin{aligned}
\operatorname{Pr}\{F(t)\} / \operatorname{Pr}\left\{H\left(t^{\prime}\right)\right\} & =f(t) / \int_{0}^{t^{\prime}} f(s) d s \\
& =\frac{1}{t^{\prime}}\left(1+\frac{f^{\prime}(0)}{f(0)}\left(t-t^{\prime} / 2\right)\right)+O\left(t^{\prime}\right),
\end{aligned}
$$

which is true if $f$ is smooth and $f(0)$ is not zero. We see that $G\left(t, t^{\prime}\right) \cap H\left(t^{\prime}\right) \subseteq E(0)$. Hence certainly

$$
G\left(t, t^{\prime}\right) \cap F(t) \cap H\left(t^{\prime}\right) \subseteq E(0)
$$

Hence

$$
\operatorname{Pr}\left\{G\left(t, t^{\prime}\right) \cap F(t) \cap H\left(t^{\prime}\right) \cap E(0)\right\}=\operatorname{Pr}\left\{G\left(t, t^{\prime}\right) \cap F(t) \cap H\left(t^{\prime}\right)\right\}
$$

Thus

$$
\begin{aligned}
\operatorname{Pr}\left\{G\left(t, t^{\prime}\right) \cap F(t) \cap H\left(t^{\prime}\right) \mid E(0)\right\} & :=\operatorname{Pr}\{E(0)\}^{-1} \operatorname{Pr}\left\{G\left(t, t^{\prime}\right) \cap F(t) \cap H\left(t^{\prime}\right) \cap E(0)\right\} \\
& =\mu\left(z(0), t_{0}\right)^{-1} \operatorname{Pr}\left\{G\left(t, t^{\prime}\right) \cap F(t) \cap H\left(t^{\prime}\right)\right\} .
\end{aligned}
$$

Let $\operatorname{Pr}_{H^{\prime}}\{$.$\} denote the conditional probability of an event, given H\left(t^{\prime}\right)$. Then from what we have just seen,

$$
\begin{aligned}
\operatorname{Pr}_{H^{\prime}}\left\{G\left(t, t^{\prime}\right) \cap F(t) \mid E(0)\right\} & :=\operatorname{Pr}\left\{G\left(t, t^{\prime}\right) \cap F(t) \cap H\left(t^{\prime}\right) \mid E(0)\right\} \operatorname{Pr}\left\{H\left(t^{\prime}\right)\right\}^{-1} \\
& =\operatorname{Pr}\left\{G\left(t, t^{\prime}\right) \cap F(t) \cap H\left(t^{\prime}\right)\right\} \mu^{-1} \operatorname{Pr}\left\{H\left(t^{\prime}\right)\right\}^{-1}
\end{aligned}
$$

Now,

$$
\int_{0}^{\infty} d t^{\prime} \int_{0}^{t^{\prime}} d t \operatorname{Pr}_{H^{\prime}}\left\{F(t) \cap G\left(t, t^{\prime}\right) \mid E(0)\right\}=1 .
$$

as the last collision must have happened for some $t$ and some $t^{\prime}>t$. Hence

$$
\int_{0}^{\infty} d t^{\prime} \int_{0}^{t^{\prime}} d t \operatorname{Pr}\left\{G\left(t, t^{\prime}\right) \cap F(t) \cap H\left(t^{\prime}\right)\right\} \mu^{-1} \operatorname{Pr}\left\{H\left(t^{\prime}\right)\right\}^{-1}=1 .
$$


As $\mu\left(z(0), t_{0}\right)$ does not depend on $t$ or $t^{\prime}$, we get

$$
\mu\left(z(0), t_{0}\right)=\int_{0}^{\infty} d t^{\prime} \int_{0}^{t^{\prime}} d t \frac{\operatorname{Pr}\left\{G\left(t, t^{\prime}\right) \cap F(t) \cap H\left(t^{\prime}\right)\right\}}{\operatorname{Pr}\left\{H\left(t^{\prime}\right)\right\}} .
$$

We can omit $H$ from the numerator, as $t<t^{\prime}$ is enforced by the region of integration. So

$$
\begin{aligned}
\mu & =\int_{0}^{\infty} d t^{\prime} \int_{0}^{t^{\prime}} d t \frac{\operatorname{Pr}\left\{G\left(t, t^{\prime}\right) \cap F(t)\right\}}{\operatorname{Pr}\left\{H\left(t^{\prime}\right)\right\}} \\
& =\int_{0}^{\infty} d t^{\prime} \int_{0}^{t^{\prime}} d t \frac{\operatorname{Pr}\left\{G\left(t, t^{\prime}\right) \mid F(t)\right\} \operatorname{Pr}\{F(t)\}}{\operatorname{Pr}\left\{H\left(t^{\prime}\right)\right\}} .
\end{aligned}
$$

Also, if $t<t^{\prime}$, then

$$
\operatorname{Pr}\left\{G\left(t, t^{\prime}\right) \mid F(t)\right\}=\bar{N} \bar{p}\left(z(t), t_{0}-t\right) w\left(z(t), t_{0}-t ; t^{\prime}\right)
$$

(what we have been calling a collision is a thermalization). In applying (21), we note that the case $f(0)=0$ corresponds to no collisions, so we may assume that $f(0) \neq 0$. We expand (23) up to first order in $t$, as $t \leq t^{\prime}$, and $t^{\prime} \leq T=20 t_{\ell}=O\left(t_{\ell}\right)$; this gives

$$
\operatorname{Pr}\left\{G\left(t, t^{\prime}\right) \mid F(t)\right\}=\bar{\mu}\left(z, t_{0}\right) w\left(z, t_{0} ; t^{\prime}\right)-t\left[\partial_{i} k_{i} / m+\partial_{0}-\left(\partial_{j} \Phi\right) \frac{\partial}{\partial k_{j}}\right] \bar{\mu}\left(z, t_{0}\right) w\left(z, t_{0} ; t^{\prime}\right) .
$$

Put this, and use (21), in (22) to get

$$
\begin{aligned}
\mu\left(z, t_{0}\right) & =\int_{0}^{\infty} d t^{\prime}\left[\bar{\mu}\left(z, t_{0}\right) w\left(z, t_{0}, t^{\prime}\right)\right. \\
& \left.-t^{\prime} / 2\left(\frac{k_{i} \partial_{i}}{m}+\partial_{0}-\left(\partial_{j} \Phi\right) \frac{\partial}{\partial k_{j}}\right) \bar{\mu}\left(z, t_{0}\right) w\left(z, t_{0} ; t^{\prime}\right)\right] .
\end{aligned}
$$

The unknown term involving $f^{\prime} / f$ does not appear, because $\int_{0}^{t^{\prime}}\left(t-t^{\prime} / 2\right) d t$ vanishes, and $\int_{0}^{t^{\prime}}\left(t-t^{\prime} / 2\right) t d t$ is of second order in $t^{\prime}$, and so can be neglected. We can now do the integral over $t^{\prime}$, using (15), and (16). This gives

$$
\mu=\bar{\mu}-\frac{1}{2}\left(\frac{k_{j} \partial_{j}}{m}+\partial_{0}-\left(\partial_{j} \Phi\right) \frac{\partial}{\partial k_{j}}\right)\left(\bar{\mu} t_{\ell}\right)
$$

This equation was derived in [7] using another method (aka guesswork), in the case when $\Phi=0$.

It does not seem fruitful to seek accuracy up to and including $O\left(t_{\ell}^{2}\right)$. This would involve introducing unknown parameters, such as $f^{\prime} / f$; worse, we would have to keep terms of second order in taking the continuum limit of the lattice; this introduces diffusion terms into the equations, which come from the Ito corrections to calculus. A similar extension of the work of Chapman and Cowling [3], who start with the Boltzmann equation rather than a master equation, is generally agreed not to have been worth the effort. Keeping only terms of first order in $t_{\ell}$ leads to the surprising result that the equations of motion are determined, knowing only that $\bar{\mu}$ is in LTE. We do not need to assume, as is done in [1], that the means of the slow variables in the approximating $L T E$-state $\bar{\mu}$ are the same as the true means, in the state $\mu$. Indeed, this turns out not to be the case; the reason is that $\bar{\mu}$ is conditioned by the fact that a thermalisation has occurred, and is not just the maximum entropy estimate of $\mu$. Then $\mu\left(\boldsymbol{x}, t_{0}\right)$ is the sum of such terms from various 
nearby points $\left(\boldsymbol{x}-\boldsymbol{k} t / m, t_{0}-t\right)$ and for a given $\boldsymbol{k}$, all the contributions are from one side of $\boldsymbol{x}$, so the means should differ unless the state is constant in space and time. In this paper we neglect the difference between $\mu$ and $\bar{\mu}$, which is the cause of dissipation in the Navier-Stokes equations. We arrive at the Euler equations, and show that they are free of dissipation, as expected.

3. The Euler equations. The Euler equations follow from the approximation of zero ${ }^{\text {th }}$ order, in which the difference between $\mu$ and $\bar{\mu}$ is neglected. Mean fields for states in LTE are computable in terms of Gaussian integrals.

The velocity field of a particle is the random field $\Upsilon_{\boldsymbol{x}}:=\mathcal{P}_{\boldsymbol{x}} / m$, and the mean current of a random variable $\chi$, conserved or not, is, in the continuum, low-density limit

$$
\mathbf{J}_{\chi}=\int d^{3} k \mu \Upsilon \Upsilon \chi
$$

Our assumption is that on thermalization there is no change in the means of $\mathcal{N}_{\boldsymbol{x}}, \mathcal{P}_{\boldsymbol{x}}$ or $\mathcal{E}_{\boldsymbol{x}}$. Since the space integrals of $N_{\boldsymbol{x}}$ and $E_{\boldsymbol{x}}$ are to be conserved in time, their equations of motion are of the form

$$
\begin{aligned}
& \dot{\rho}+\partial_{j} J_{\rho}^{j}=0 \\
& \dot{E}+\partial_{j} J_{E}^{j}=0 .
\end{aligned}
$$

Momentum is not conserved; in a small volume at $\boldsymbol{x}$ a particle of momentum $\boldsymbol{k}$ in time $d t$ is changed to $\boldsymbol{k}-\nabla \Phi d t$, so the momentum obeys

$$
\frac{d \varpi^{i}}{d t}+\partial_{j} J_{\varpi^{i}}^{j}+\frac{\rho}{m} \partial_{i} \Phi=0
$$

In [7] we showed that we can evaluate the currents (26) of hydrodynamics exactly if $\mu$ is in $L T E$, with parameters $\rho, u^{i}, \Theta$ say. By the same method, (27)-(29) can be written out:

$$
\begin{gathered}
\frac{d \rho}{d t}+\partial_{j}\left(\rho u_{j}\right)=0 \\
\frac{d}{d t}\left(\rho u_{i}\right)+\partial_{j}\left(\rho u_{i} u_{j}\right)+\partial_{i} P+\rho \partial_{i} \phi=0 \\
\frac{d}{d t}\left\{\rho\left(\frac{3 k_{B}}{2 m} \Theta+\frac{1}{2} u_{j} u_{j}+\phi\right)\right\}+\partial_{j}\left\{\rho u_{j}\left(\frac{3 k_{B}}{2 m} \Theta+P / \rho+\frac{1}{2} u_{j} u_{j}+\phi\right)\right\}=0 .
\end{gathered}
$$

Here, $\phi=\Phi / m$. Note that the pressure $P$ appearing in these equations is that of an ideal gas, not the equilibrium pressure of the model; the two differ by terms of second order in the density. This reflects that the derivation of the fundamental equation depends on the assumption that the density is small.

4. Entropy production. From von Neumann's formula,

$$
S:=-\sum_{\omega} \mu(\omega) \log \mu(\omega)
$$

we conclude that

$$
\dot{S}=-\sum_{\omega} \dot{\mu}(\omega) \log \mu(\omega)-\sum_{\omega} \dot{\mu}(\omega)
$$


The last term is zero, so we may regard $-\log \mu$ as a random variable $\mathcal{S}$ whose average rate of change is given in the Schrödinger picture by $\dot{\mu} \cdot \mathcal{S}$. The current of the entropy would then be, per site,

$$
a^{3} \mathcal{J}_{S}^{j}(x)=\mathcal{S}(\omega) \Upsilon^{j}=\xi \mathcal{N} \Upsilon^{j}+\beta \mathcal{E} \Upsilon^{j}+\zeta^{i} \mathcal{P}_{i} \Upsilon^{j}+\log \Xi \Upsilon^{j}
$$

when $\mu=N p$ and $p$ is in LTE.

We must clarify the definition of $\Upsilon^{i}(\omega)$ when $\omega=\emptyset$, as zero is not a good definition; the information in there being no particles at $\boldsymbol{x}$ is carried along with the general fluid. Let us define $\Upsilon^{i}(\emptyset):=u^{i}$ when the state $\mu$ has velocity $u^{i}$. In that case, the mean of $\Upsilon^{i}$ in the state $\mu$ is

$$
\mu(\emptyset) u^{i}+\sum_{k} N p \cdot \Upsilon^{i}=(1-N) u^{i}+N u^{i}=u^{i} .
$$

The mean density of the entropy current, $J_{S}^{j}$, in the state $\mu$ then has three terms:

$$
\begin{aligned}
\xi a^{-3} \mu \cdot\left(\mathcal{N} \Upsilon^{j}\right)+a^{-3} \log \Xi \mu \cdot \Upsilon^{j} & =\frac{\xi}{m} J_{\rho}^{j}-\log (1-N) u^{j}, \\
a^{-3} \zeta^{i} \mu \cdot\left(\mathcal{P}_{i} \Upsilon^{j}\right) & =\zeta^{i} J_{\varpi_{i}}^{j}, \\
a^{-3} \beta \mu \cdot\left(\mathcal{E} \Upsilon^{j}\right) & =\beta J_{E}^{j} .
\end{aligned}
$$

We expect that entropy will be conserved, because of Kossakowski's argument [4]: in the absence of collisions, the projections onto the information manifold do not create any entropy if time is continuous. See also [2]. Without collisions, Boltzmann's equation also gives zero entropy production, if the assumption of $L T E$ is made. So we expect that the rate of change of entropy is balanced by the outflow though the boundary:

$$
\dot{s}+\partial_{j}\left(\frac{\xi}{m} J_{\rho}^{j}+\zeta^{i} J_{\varpi_{i}}^{j}+\beta J_{E}^{j}-\log (1-N) u^{j}\right)=0 .
$$

where $s$ denotes the entropy density. On the other hand, we have

$$
a^{3} s=\xi N+\zeta^{i} \Pi_{i}+\beta E+\log \Xi,
$$

giving

$$
\begin{aligned}
a^{3} \frac{d s}{d t}= & N \partial_{t} \xi+\Pi_{i} \partial_{t} \zeta^{i}+E \partial_{t} \beta+\frac{1}{\Xi}\left(\frac{\partial \Xi}{\partial \xi} \partial_{t} \xi+\frac{\partial \Xi}{\partial \zeta^{i}} \partial_{t} \zeta^{i}+\frac{\partial \Xi}{\partial \beta} \partial_{t} \beta\right) \\
& +\xi \partial_{t} N+\zeta^{i} \partial_{t} \Pi_{i}+\beta \partial_{t} E
\end{aligned}
$$

Now,

$$
\frac{1}{\Xi} \frac{\partial \Xi}{\partial \xi}=-N \text { etc. }
$$

so the first line (37) vanishes, to leave

$$
a^{3} \partial_{t} s=\xi \partial_{t} N+\zeta^{i} \partial_{t} \Pi_{i}+\beta \partial_{t} E .
$$

We divide by $a^{3}$ to get the densities $\rho, a^{-3} E$ and $\varpi_{i}$, and the relation

$$
\int_{\Lambda} \partial_{t} s d^{3} x=\int_{\Lambda}\left(\frac{\xi}{m} \partial_{t} \rho+\zeta^{i} \partial_{t} \varpi_{i}+\beta \partial_{t} E / a^{3}\right) d^{3} x
$$




$$
\begin{aligned}
& =-\int_{\Lambda}\left(\frac{\xi}{m} \partial_{j} J_{\rho}^{j}+\zeta^{i}\left(\partial_{j} J_{\varpi_{i}}^{j}+\rho \delta_{i j} \partial_{j} \phi\right)+\beta \partial_{j} J_{E}^{j}\right) \\
& =\int_{\Lambda}\left(\zeta^{i} \rho f_{i}+\partial_{j} \xi J_{\rho}^{j}+\partial_{j} \zeta^{i} J_{\varpi_{i}}^{j}+\partial_{j} \beta J_{E}^{j}\right) \\
& -\oint_{\partial \Lambda}\left(\xi J_{\rho}^{n}+\zeta^{i} J_{\varpi_{i}}^{n}+\beta J_{E}^{n}\right) d \sigma_{n}
\end{aligned}
$$

where $n$ denotes the normal direction to the boundary. The term in (41) involving $f_{i}=$ $-\partial_{i} \Phi / m$ represents the free-energy change due to the work done by the external field on the particles; we shall see that this is cancelled out by another term, showing that this work done is not thermalised by the dynamics. In spite of the apparent Onsager form of (40), we cannot identify it as the entropy production and (41) as the flow through the boundary, because the surface term differs from the entropy current, as in (36) by $-\log (1-N) u^{j}$. Indeed, if we put the Euler currents in (40) we do not get zero, even when $\Phi=0$. We must therefore add and subtract the missing term, to get

$$
\begin{aligned}
\partial_{t} s= & \int_{\Lambda}\left(\zeta^{i} \rho f_{i}+\partial_{j} \xi J_{\rho}^{j}+\partial_{j} \zeta^{i} J_{\varpi_{i}}^{j}+\partial_{j} \beta J_{E}^{j}-\partial_{j}\left(a^{-3} \log (1-N) u_{j}\right)\right) d^{3} x \\
& -\oint_{\partial \Lambda} J_{S}^{i} d \sigma_{i} .
\end{aligned}
$$

If we now put in the Euler currents, (30)-(32), we still do not find exactly zero for the entropy production, (42). Indeed, the entropy production involves the logarithm, whereas the Euler equations are polynomial, so cancellation is not possible. We find that (40) vanishes up to terms $O\left(N^{2}\right)$, that is, in the limit of low density. This reflects the low-density assumption inherent in the axiom that the $B B G K Y$ currents, (26), are the actual currents of particles flowing out of the region $d^{3} x$. While this is a reasonable model for point-like particles, it neglects the fact that if the lattice-site just beyond the boundary is occupied, then a particle moving out of the region will not be able to land, as no configuration in the sample space can have two particles at the same site. Our dynamics did not need to specify the rule as to what will happen, as (in the low density limit) the probability that the point is occupied is small.

It turns out that if we modify the pressure in the Euler equations, to be the equilibrium pressure of the gas with hard core, (10), rather than that of the ideal gas, then the entropy production is exactly zero, in conformity with Kossakowski's theorem:

TheOREm 1. Take the pressure $P$ in (31) and (32) to be that of the hard-core gas at equilibrium, (10). Then the entropy production, (42), is zero.

Proof. We first recall the relation between the canonical variables and the hydrodynamic variables. We saw that

$$
\beta=\frac{1}{k_{B} \Theta}, \quad \zeta^{j}=-\beta u^{j}=-\frac{u^{j}}{k_{B} \Theta},
$$

so from (7) we get for the entropy production (42):

$$
\begin{aligned}
\dot{s}_{\text {prod }} & =\zeta^{i} \rho f_{i} \\
& +\frac{\rho u^{j}}{m}\left(\frac{3}{2} \frac{\partial_{j} \Theta}{\Theta}-\frac{m}{2 k_{B}} \frac{u^{i} u^{i}}{\Theta^{2}} \partial_{j} \Theta+\frac{m}{k_{B}} \frac{u^{i}}{\Theta} \partial_{j} u^{i}-\frac{\partial_{j} N}{N}-\frac{\partial_{j} N}{1-N}-\frac{m}{k_{B}} \frac{\partial_{j} \phi}{\Theta}+\frac{m}{k_{B}} \frac{\phi}{\Theta^{2}} \partial_{j} \Theta\right)
\end{aligned}
$$




$$
\begin{aligned}
& +\left(\rho u_{i} u_{j}+\delta_{i j} P\right)\left(-\frac{\partial_{j} u^{i}}{k_{B} \Theta}+\frac{u^{i}}{k_{B}} \frac{\partial_{j} \Theta}{\Theta^{2}}\right)+\rho u_{j}\left(\frac{3 k_{B}}{2 m} \Theta+\frac{P}{m}+\frac{1}{2} u^{i} u^{i}+\phi\right) \frac{-\partial_{j} \Theta}{k_{B} \Theta^{2}} \\
& +a^{-3} \frac{\partial_{j} N}{1-N} u^{j}-a^{-3} \log (1-N) \partial_{j} u^{j} \\
& =0 .
\end{aligned}
$$

Acknowledgements. This work summarizes my contributions to the conference on "Nonlocal Elliptic and Parabolic Problems", Będlewo, and to the meeting of the Polish Physical Society, Gdańsk, 2003. I thank P. Biler, G. Karch, T. Nadzieja, R. Alicki and W. A. Majewski for arranging the visits.

\section{References}

[1] R. Balescu, Statistical Dynamics, Imperial College Press, 1997.

[2] R. Balian, Y. Alhassid, and H. Reinhardt, Dissipation in many-body systems, Phys. Rep., 131 (1986), 2-101.

[3] S. Chapman and T. G. Cowling, The Mathematical Theory of Non-Uniform Gases, $3^{\text {rd }}$ ed., Cambridge University Press, 1970.

[4] A. Kossakowski, On the quantum informational dynamics, Bull. Acad. Pol. Sci. Sér. Sci. Math. Astronom. Phys. 17 (1969), 263-269.

[5] P. L. Lions, Mathematical Topics in Fluid Dynamics, I, II, Clarendon Press, Oxford, 1996.

[6] J.-P. Rivet and J. P. Boon, Lattice Gas Hydrodynamics, Cambridge University Press, 2001.

[7] R. F. Streater, Corrections to Fluid Dynamics, Open Systems and Information Dynamics, 10 (2003), 3-30. arXiv/math-ph/0105013 